\title{
AN INTERESTING CASE OF HUGE COLD ABSCESS CAUSING RESPIRATORY DISTRESS
}

Sudhir Bhargava ${ }^{1}$, Suresh Rathi ${ }^{2}$

\section{HOW TO CITE THIS ARTICLE:}

Sudhir Bhargava, Suresh Rathi."An Interesting Case of Huge Cold Abscess Causing Respiratory Distress”. Journal of Evolution of Medical and Dental Sciences 2014; Vol. 3, Issue 46, September 22; Page: 11288-11291, DOI: $10.14260 /$ jemds/2014/3472

ABSTRACT: A rare case of an adult suffering with potts spine and a huge retropharyngeal cold abscess completely obstructing the upper airway and larynx, causing respiratory distress is presented as an acute emergency. An urgent tracheostomy to safeguard the airway was required. The clinical features, surgical anatomy, aetiopathogenesis and management of a retropharyngeal abscess are discussed.

KEYWORDS: Cold Abscess.

INTRODUCTION:A collection of pus behind the posterior pharyngeal wall is called a retropharyngeal abscess. The prevertebral fascia separates the visceral compartment of the neck containing the pharynx,trachea, larynx and the thyroid gland from the cervical vertebrae and the prevertebral muscles. The buccopharyngeal fascia covers the side and the back of the pharynx.The prevertebral fascia is firmly attached at the midline of the back of the pharynx.

The potential space in between the prevertebral fascia and the buccopharyngeal fascia is known as the retropharyngeal space or also known as the space of Gillette. These spaces are separated from each other by a firm attachment of the above mentioned fasciae. The retropharyngeal space contains prevertebral or the retropharyngeal lymph nodes.Children over the age of 4 years have far fewer glands in this space following their atrophy; hence the infection is more common in very young children. ${ }^{1}$

A retropharyngeal abscess may be acute, chronic or traumatic in nature/ origin. A retropharyngeal abscess is likely to be due to tuberculous infection of the spine which is common most site of the skeletal tuberculosis. The body of the vertebra is most commonly affected by the blood-borne infection ${ }^{2}$. It is of slow origin and it gives rise to pharyngeal discomfort rather than pain and to some degree of dysphagia. ${ }^{3}$

The chronic abscess is due to the caries of the cervical spine the patient is adolescent the abscess is median in position and is unassociated with the toxemia. An attitude of supporting head with the hands,rigidity and x-ray neck are diagnostic 4 .The case is a documented because of the rarity of reports on retropharyngeal abscess in the literature and because of the large size of a retropharyngeal cold abscess sufficient to cause respiratory distress in an adult patient.

CASE REPORT:A 34 years old male presented with a 15 days history of difficulty in breathing, stiff neck, dysphagia, cough and low grade pyrexia. On examination of the throat revealed a diffuse retropharyngeal smooth swelling in the oropharynx. It extends towards the laryngopharynx and its lower limit was not visible. $X$-rays of the neck showed a huge soft tissue shadow with the destruction of the5th and the $6^{\text {th }}$ cervical vertebrae; a chest x-ray was normal.(See picture 1.of x-ray cervical spine lateral view.). 
Lateral X-ray neck showing huge retropharyngeal abscess with tuberculous spine (c5, c6)obstructing the larynx.

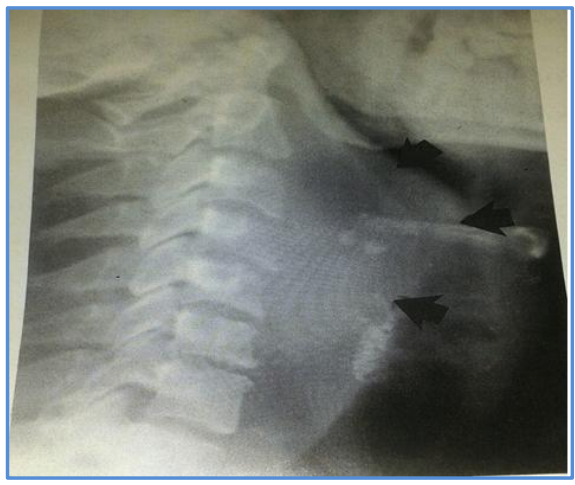

Fig. 1

An urgent tracheostomy was performed under the local anesthesia to relieve the patient of the respiratory distress.A provisional diagnosis of the tuberculous disease of the spine with a retropharyngeal abscess was made and immediate antituberculous medication was started with injection streptomycin 1gm intramuscularly,tab rifampicin 450mgm,INH 300 milligram and tab ethambutol 800 milligram orally per day.

OPERATIVE PROCEDURE: Transoral aspiration of the retropharyngeal abscess was carried out 48 hours after presentation using a long wide bore needle under the local anesthesia after opening the mouth by inserting Boyles Davis mouth gag.100 ml of thick yellow pus was aspirated.Similar aspirations were carried out at 3-4 days intervals. The amount of pus aspirated gradually decreased along with the size of the retropharyngeal abscess.

Seven such aspirations were done in all over the period of 3 weeks. All the samples aspirated showed acid-fast gram negative bacilli,thus confirming our clinical diagnosis of Potts disease of spine associated with a huge retropharyngeal abscess.At follow up nearly six 3 months later Xray of his cervical spine showed a near normal prevertebral space and the patient was given antitubercular treatment for 15 months.(See picture 2 x-ray cervical spine lateral view showing nearly normal retropharyngeal space).

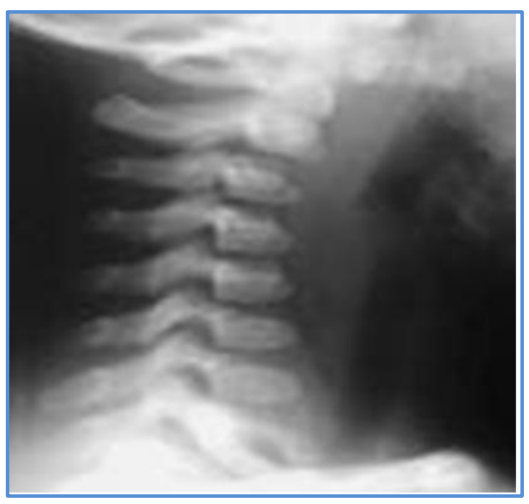

Fig. 2 
DISCUSSION:Retropharyngeal abscess may be acute, chronic or traumatic in origin caused by a perforating foreign body. Acute RPA occurs commonly in children and is caused by suppuration in a retropharyngeal lymph node following an upper respiratory tract infection.It occurs in front of the prevertebral fascia,but on one side of the midline because of the attachment of the buccopharyngeal fascia to the prevertebral fascia.It is seen mostly in children because the glands of Gillette disappear before the age of 5 years. 3,5

The chronic type of RPA forms due to tuberculosis of the cervical vertebra and is therefore behind the prevertebral fascia,central in midline position and it bulges into the pharynx in midline. Any one or several vertebrae may be involved by the disease.The infection starts in the cancellous area of the vertebral body where it may be mainly central,anterior or epiphyseal in location.

Infection of the posterior bony arch and the transverse process is unusual.6In tuberculosis of the cervical vertebrae,debris frequently accumulates which forms a collection of fluid known as a cold abscess because it is devoid of acute symptoms such as pain, fever, and swelling. The exudates in a cold abscess is composed of leucocytes, caseous material, bone and the tubercle bacilli. ${ }^{6}$

As a result of the disease one or more of the bodies of the vertebrae may be involved to the point where they can no longer support the weight transmitted through them and they consequently collapse.The debris in their interior is squeezed out by this collapse and travels along the paths which are determined by the surrounding anatomy,presenting as a COLD ABSCESS.

In the cervical region the abscess may bulge in to the mouth or the pharynx but can also appear at the posterior border of the stern mastoid muscle, or it may descend by tracking downwards behind the prevertebral fascia and guided by it into the posterior mediastinum. A cold abscess may also appear at the back of the neck or in the axilla as the pus extends from its site of origin along the path of least resistance guided by through facial attachments. ${ }^{7}$

The most reliable clinical method of establishing the diagnosis is a lateral neck x-ray.Normally the anteroposterior diameter of the prevertebral soft tissue shadow should not exceed that of the vertebral bodies. Computed tomography scanning has also been shown to be of value, especially in assessing the extent of the abscess cavity ${ }^{8}$.This may also makes it easier to decide which route to use for the drainage. X-rays however, must be interpreted correctly and a true lateral x-ray is necessary. ${ }^{9}$

In the case presented, lateral x-ray of the neck was diagnostic of the pott's disease of the cervical spine with a huge retropharyngeal abscess. This was confirmed by the presence of the acidfast bacilli in the aspirate and by the patient response to the antituberculous régime. In tuberculous caries of the spine with a retropharyngeal abscess,various combinations of STREPTOMYCIN,PAS,INAH and other more recent ant tubercular drugs are used. Surgery is indicated only when there is embarrassment to respiration or swallowing.

The abscess may be drained either via a transoral approach or through the neck.The accepted method is an aspiration and by an incision at the most convex point by the external approach.The abscess is drained in most instances by an incision in front of the stern mastoid muscle,in the plane between the carotid sheath and the visceral compartment of the neck if the abscess is high in the neck.It is better approached from behind the carotid sheath through an incision behind the stern mastoid muscle. 10 


\section{REFERENCES:}

1. Grodinski.M. Hoiyoke.e.a.l(1958).The fascia and the facial spaces of the head and neck and adjacent region American journal of anatomy 63; 367-408.

2. Mcgregor. AL. (1966) A synopsis of the surgical anatomy $12^{\text {th }}$ edition (1957)p 206-65.

3. Turner (1956) Logan turner diseases of ENT $9^{\text {th }}$ edition $\mathrm{p}$ 202-204.

4. Bhargava kb et al pharyngeal abscesses a short text book of ENT diseases $4^{\text {th }}$ edition $\mathrm{p}$ 296-304.

5. Adams \& Daw (1962) retropharyngeal cellulites following a wound injury of the pharynx JLO 79; 559-662.

6. Turek i. (1984) ortho principles and their application 4th vol edition $2^{\text {nd }}$ p 1577.

7. Ballantyne JC (1086) surgical treatment of the parapharyngeal abscess in Robs andSmith op ENT $4^{\text {the }}$ p 203-205.

8. White B.(1985). Deep neck infection and the respiratory distress in ENT journal 64; 30-38.

9. Said ab Dunbar etl (1979) retropharyngeal abscess laryngoscope 89; 1717-1724.

10. Das k. (19810 Clinical methods in surgery $11^{\text {the }}$ dition 191-194.

\section{AUTHORS:}

1. Sudhir Bhargava

2. Suresh Rathi

\section{PARTICULARS OF CONTRIBUTORS:}

1. Senior ENT Consultant \& Laser Surgeon, Department of ENT, CHL-HOSPITALS, Indore(MP)

2. Consultant Surgeon, Department of General Surgery, Medisqure Hospital, Indore.

\section{NAME ADDRESS EMAIL ID OF THE CORRESPONDING AUTHOR:}

Dr. Sudhir Bhargava,

120, Prakash Nagar,

Navlakha, Indore,

Madhya Pradesh.

Email: sudhir_laser@yahoo.com

Date of Submission: 05/09/2014.

Date of Peer Review: 06/09/2014.

Date of Acceptance: 15/09/2014.

Date of Publishing: 22/09/2014. 